\title{
Primeiras observações sobre o Aedes Albopictus no Estado do Espirito Santo, Brasil.
}

*SUCAM, Caixa Postal, 190, 88.000 Florianopolis, SC.

*FIocruz, Caixa Postal. 926, 20010 Rio de Janeiro, RJ.

\author{
Joaquin A. Ferreira Neto : \\ Milton Moura Lima** \\ Mario B. Aragão**
}

Observações realizadas no Estado do Espirito Santo mostraram que o Aedes albopictus, ao ser introduzido no Brasil, não modificou, significativamente, o seu comportamento. Os criadouros são os mesmos u tilizados na Asia e situados, de preferência, em áreas abertas. Os mosquitos adultos se abrigam em vegetação baixa e freqüentam pouco o domicilio.

Algumas larvas encontradas num pneu de trator, abandonado debaixo de arbustos, na Universidade Rural (Município de Itaguaí. Estado do Rio de Janeiro) foram criadas pelo Prof. Eugênio Izeckson e identificadas como Aedes albopictus, pela primeira vez no Brasil, pelo Prof. Forattini (Forattini). ${ }^{4}$

Um levantamento preliminar realizado pela Superintendência de Campanhas de Saúde Pública (SUCAM) revelou uma grande dispersão da espécic no Estado do Espírito Santo, praticamente toda a metade sul do Estado. Por esse motivo, foram feitas algumas observaçōes sobre 0 comportamento da espécie, nessa sua nova pátria que. acrescidas das informações disponive is na bibliografia. constituem o objeto de sta publicação.

O $A$. albopictus é um mosquito originário do sudeste da Ásia e tudo indica ser, primariamente, silvestre. Nessa região ele é comum nos restos de floresta, ainda existentes, nos bosques de bambu e nos coqueirais, mas também ocorre nas áreas rurais e suburbanas. O seu criadouro primitivo é o buraco de pau mas, mesmo no sudeste da Ásia ele se cria em pneus e $\mathrm{cm}$ latas.

Gilotra el al. trabalhando em Calcutá, que por sinal é a localidade típica da espécie, dizem que os habjtats mais típicos são os bosques de bambu e os coqueirais, mas que cle invade o ambiente doméstico. Neste, usa os mesmos depósitos artiliciais utilizados pelo Aceles aegypti, preferindo porem os siluados cm volta das casas. Chan et al. (1971) mencionam como principais criadouros, en (in) gapura, poles de barro, armadilhas de formiga, pneus, tinas e tambores. Desses, $50 \%$ situam-se fora de casa.

No Japão, onde a espécic é in troduzida, se cria em to- 
dos os depósitos de água limpa, situados à sombra ou à meia sombra. ${ }^{6}$ Nesse país ele também é encontrado em florestas, porém, para Mogy (1982), essa adaptação é secundária.

$\mathrm{Na}$ itha de Guam, onde a espécie foi descoberta em 1944, já em 1948 estava bem distribuída e era encontrada em buracos de pau, bambu, cascas de coco e bracteas de palmeira (Rozeboom \& Bridges, 1972).

Em Honolulu, no Havai, a espécie é recente, mas, em algumas épocas já foi mais numerosa do que o $A$. aegypti. ${ }^{9}$ Nessa cidade os principais cria douros são latas, vasos de flo. res e uma planta da família das liliáceas. ${ }^{1}$

Sobre o comportamento do A. albopictus nas Américas não existem informações. Sabe-se que ele foi de tectado nos Estados Unidos em 1985 e já está bem espalhado no sul daquele país. ${ }^{2}$

No Espiríto Santo o $A$. albopictus tem sido encon tra. do em alta densidade nos depósitos de pneus usados, onde se abriga na vegetação baixa, situada ao redor (Figura 1). Já no Município de Anchieta tem sido coletado em capoeiras baixas (Figura 2), porém, os criadouros só foram encon. trados fora dessa vegetação.

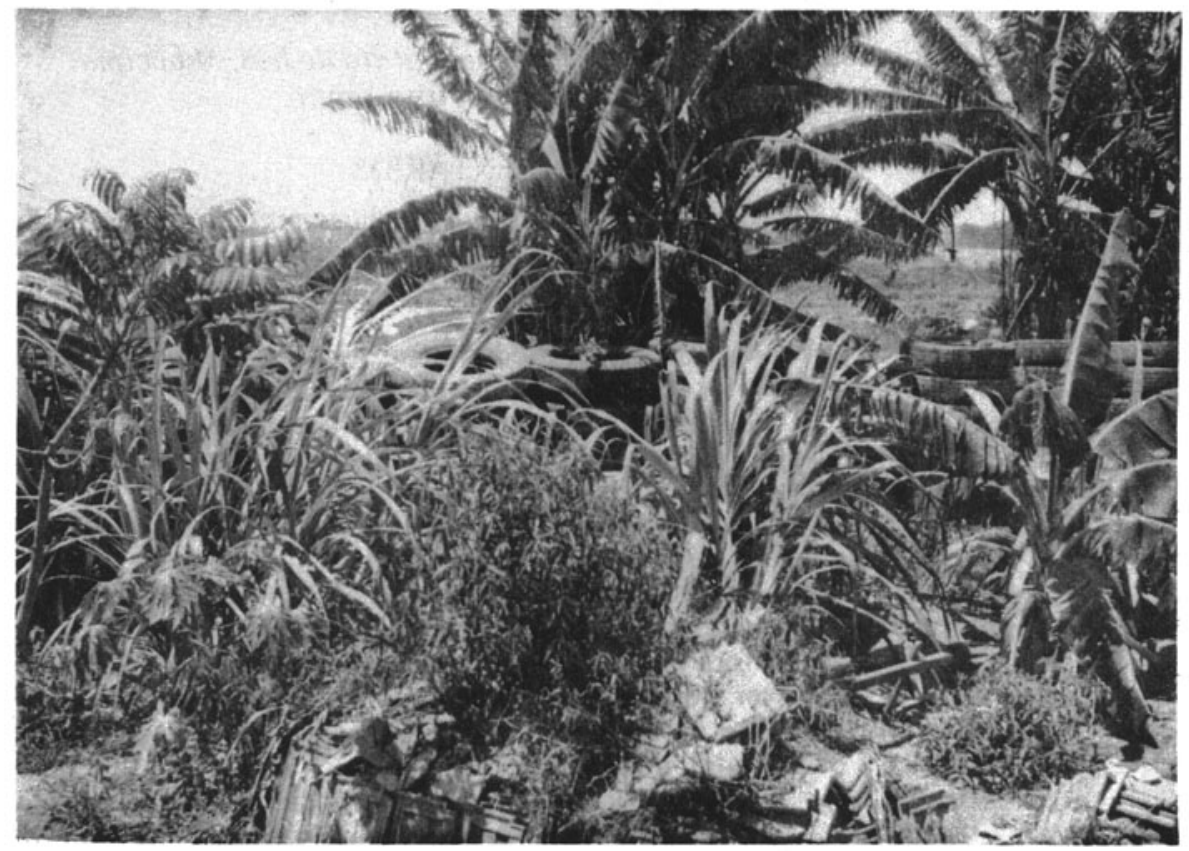

Figura 1 - Vegetaçāo baixa em torno de um depósito de pneus usados, no Municipio de Serra, Espirito Santo.

Cadernos de Saúde Pública, RJ, 3 (1): 56-61, jan/fev, 1987 


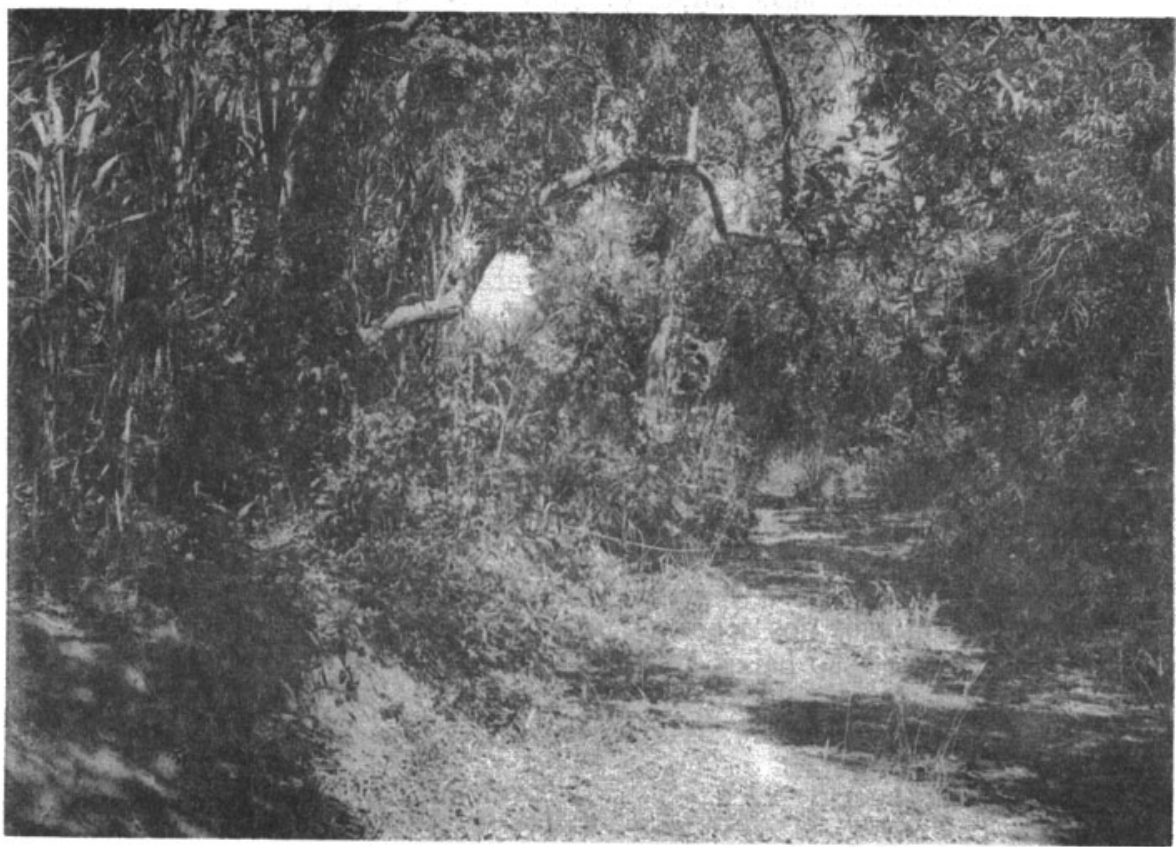

Figura 2 - Capoeira rala no Distrito de Iriri, Municipio de Anchieta, Espirito Santo.

\section{RESULTADOS E COMENTÁRIOS}

De um modo geral, os índices prediais encontrados têm sido baixos (Tabela 1). Apenas no Distrito de Iriri, no Município de Anchieta, esse índice ultrapassou a $10 \%$ (Tabela 2).

Os criadouros predominam fora das casas e na tabela 1 , pode ser visto que eles não são raros nos terrenos baldios.

$\mathrm{Na}$ tabela 3 estão os depósitos pesquisados no Municípjo de Anchieta e, entre eles, destacam-se os pneus. A porcentagem de poços e cisternas positivos é elevada, porém, - número pesquisado foi pequeno. Os outros depósitos grandes, como caixas dágua, barris etc também são procu. rados, porém, em porcentagem mais baixa. Sob a rubrica de depósitos diversos estão, na sua maior parte, objetos jogados fora nos quintais das casas e que, na verdade, deveriam ter sido removidos como lixo. Dentre as plantas positi. vas, há a destacar uma bromeliácea colocada, como planta ornamental, numa praça pública.

As cap turas de alados (Tabela 4) mostram que o mosquito não está preferindo o domicilio, observação que já tinha sido feita no local onde foi tirada a foto da figura 1. Em qualquer ponto protegido do vento, os mosquitos a tacavam mas não penetravam numa casa situada ao lado e onde havia pessoas. 


\section{TABELA 1}

Resultado da pesquisa de Aedes albopictus em quatro municipios do Estado do Espirito Santo, Brasil.

\begin{tabular}{lrrrrrr}
\hline \multirow{2}{*}{ Município } & \multicolumn{3}{c}{ Prédios } & \multicolumn{3}{c}{ Terrenos baldios } \\
\cline { 2 - 7 } & Insp. & pos. & $\%$ & Insp. & Pos. & $\%$ \\
\hline Vitória & 19.029 & 835 & 4,4 & 512 & 14 & 2,7 \\
Vilha Velha & 16.597 & 768 & 4,6 & 1.093 & 9 & 0,8 \\
Serra & 13.487 & 493 & 3,7 & 549 & 7 & 1,3 \\
Itapemirim & 2.409 & 58 & 2,4 & 146 & 2 & 1.4 \\
Total & 51.522 & 2.154 & 4,2 & 2.300 & 33 & 1,4 \\
\hline
\end{tabular}

Nota Insp. $=$ Inspecionados; Pos. $=$ Positivos; $\%=$ Porcentagem de positivos.

\section{TABELA 2}

Resultado da pesquisa de Aedes albopictus no Municipio de Anchieta, Estado do Espirito Santo, Brasil.

\begin{tabular}{lrrr}
\hline & \multicolumn{3}{c}{ Prédios } \\
& Insp. & Pos. & $\%$ \\
\hline Anchieta e arredores & 1.623 & 95 & 5,9 \\
Distrito de Iriri & 484 & 52 & 10,7 \\
Municipio de Anchieta & 2.107 & 147 & 7,0 \\
\hline
\end{tabular}

Nota: Insp. = Inspecionados; Pos. = Positivos; $\%=$ Porcentagem de positivos.

$\mathrm{Na}$ capoeira (Tabela 4) a densidade era alta, apesar de não terem sido encontrados criadouros dentro dela. Os depósitos positivos estavam fora.

\section{CONCLUSÕES}

A expansão da área de ocorrência do $A$. albopictus no Espírito Santo mostra que ou a invasão é antiga ou ele se dispersa muito rapidamente, como já tinha sido observado na ilha de Guam. ${ }^{3}$

Cadernos de Saúde Pública, RJ., 3 (1): 56-61, jan/tev, 1987 
TABELA 3

Resultado da pesquisa de Aedes albopictus, em depósitos encontrados no Municipio de Anchieta, Espirito Santo, Brasil.

\begin{tabular}{|c|c|c|c|c|c|c|c|c|c|c|c|c|c|c|c|c|c|c|}
\hline \multirow{2}{*}{$\frac{\text { Depósitos }}{\text { Localidades }}$} & \multicolumn{3}{|c|}{ Caixas d'água } & \multicolumn{4}{|c|}{ Barris, toneis e tinas } & \multicolumn{4}{|c|}{ Depósitos diversos* } & \multirow{2}{*}{$\frac{\text { Preus }}{\%}$} & \multicolumn{3}{|c|}{ Árores e plantas } & \multicolumn{3}{|c|}{ Poços e cisternas } \\
\hline & Insp. & Pos & $\%$ & Insp. & Pos. & $\%$ & Insp. & Pos. & $\%$ & Insp. & Pos. & & Insp. & Pos. & $\%$ & Insp. & Pos. & $\%$ \\
\hline $\begin{array}{l}\text { Cidade de Anchieta } \\
\text { e seus bairros } \\
\text { Distrito de Iriri } \\
\text { Todo Municipio de }\end{array}$ & $\begin{array}{c}1594 \\
498\end{array}$ & $\begin{array}{r}33 \\
7\end{array}$ & $\begin{array}{l}2,1 \\
1,4\end{array}$ & $\begin{array}{r}1687 \\
527\end{array}$ & $\begin{array}{r}20 \\
8\end{array}$ & $\begin{array}{l}1,2 \\
1,5\end{array}$ & $\begin{array}{l}800 \\
369\end{array}$ & $\begin{array}{r}10 \\
8\end{array}$ & $\begin{array}{l}1,3 \\
2,2\end{array}$ & $\begin{array}{l}512 \\
132\end{array}$ & $\begin{array}{l}72 \\
19\end{array}$ & $\begin{array}{l}14,1 \\
14,4\end{array}$ & $\begin{array}{r}2597 \\
744\end{array}$ & $\begin{array}{l}99 \\
43\end{array}$ & $\begin{array}{l}3,8 \\
5,8\end{array}$ & $\begin{array}{r}89 \\
-12\end{array}$ & $\begin{array}{r}11 \\
5\end{array}$ & $\begin{array}{l}12,4 \\
41,7\end{array}$ \\
\hline Anchieta & 2092 & 40 & 1,9 & 2214 & 28 & 1,3 & 11,69 & 18 & 1,5 & 644 & 91 & 14,1 & 3341 & 142 & 4,3 & 101 & 16 & 15,8 \\
\hline
\end{tabular}

-Depósitos diversos = Gerrafas e copos de plístico, frascos e cacos de vidro, latas e cochos de animais.

Nora $:$ Insp. $=$ Inspecionados: Pos. $=$ Positivos; $\%=$ Porcentagem de positivos.

\section{TABELA 4}

Resultados de capturas de Aedes albopictus feitas dentro de capeira rala, nas suas margens e dentro de casa. Municptpio de Anchieta, ES, Brasil.

\begin{tabular}{rcccccc}
\hline Capturas & \multicolumn{2}{c}{$\begin{array}{c}\text { Dentro da } \\
\text { capoeira* }\end{array}$} & \multicolumn{2}{c}{$\begin{array}{c}\text { Fora da } \\
\text { capoeira * }\end{array}$} & \multicolumn{2}{c}{$\begin{array}{c}\text { Dentro de } \\
\text { casa }\end{array}$} \\
\hline Horário & Total & Por hora & Total & Por hora & Total & Por hora \\
\hline 8 às 11 & 49 & 16,3 & 10 & 3,3 & 2 & 0,7 \\
14 às 19 & 84 & 16,8 & 17 & 3,4 & 13 & 2,6 \\
Total & 133 & 16,6 & 27 & 3,4 & 15 & 1,9 \\
\hline
\end{tabular}

- As capturas dentro e fora da capoeira foram simultâneas.

Nota - Todas as capturas foram feitas por um único homem.

Por en quanto o mosquito ainda não foi capturado em floresta, porém, o seu encontro em capoeira, se bem que rala, causa preocupação. $E_{\text {que }}$ no Estado existem diversas Reservas Florestais, tan to particulares quanto do governo, que, por lei, são áreas intocáveis. Sendo assim, seria prudente erradicar a espécie dos municípios onde se situam essas Reservas e, depois disso, manter uma vigilância rigorosa .

De um modo geral, o que tem sido observado no Espirírito Santo não difere muito do que está registrado para a sua área de origem ou de outros locais da Ásia para onde a espécie foi transportada. Criadouros, de preferência, situados fora das casas, porém, com alguma sombra, abrigo em vegetação baixa ou, quando muito, capoeira rala e pouca frequêencia ao domiculio.

\section{AGRADECIMENTOS}

Os autores são gratos ao Dr. Danilo Mauricio Cosmo, Diretor Regional da SUCAM no Espírito Santo, pelas faci- 
lidades proporcionadas ao trabalho e, também, por ter permitido a utilização de dados levantados sob sua orientação, pela SUCAM.

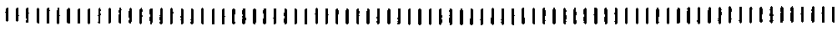

First observations on Aedes albopictus in the State of Espirito Santo, Brasil.

Observations performed at the Sta te of Espirito Santo showed that Aedes albopictus did not change its behaviour to a significant extent after being introduced in Brazil. The breeding places are the same as those described in Asia and can be found more frequently in open areas. The adult mosquitoes rest in short vegetation and only sporadically penetrate in to the human habitations.

\section{REFERÊNCIAS B IB LIOGRÄFICAS}

1. BONNET, D.D. The distribution of mosquito breeding by type of container in Honolulu, T.H. Proc. Hawn. Ent. Soc., 13: 43-9, 1947.

2. CENTER FOR DISEASE CONTROL Aedes albopictus infestation - United States, Brazil. MMWR, 35: 493-5, 1986.

3. CHAN, K.L, HO, B.C. \& CHAN, Y.C. Aedes aegypti (L.) and Aedes albopitous (Skuse) in Singapore City. 2. Larval habita ts. Bull. Wld Hlth Org., 44: 629-33, 1971.

4. FORATTINI, O.P. Identificação de Aedes (Stegomyia) albopictus (Skuse) no Brasil. Rev. Saide publ., S. Paulo, 20: 244-5, 1986.

5. GILOTRA, S.K., ROZEBOON, L.E. \&BHATTACHARYA, N.C. Observations on possible competitive displacement between populations of Aedes aegypti Lineaus and Aedes albopictus Skuse in Calcu ta. Bull. Wld Hlth Org., 37: 43746, 1967.

6. GRAG, H.F. Mosquito control problems in Japan. Mosq. News, 7: 7-11, 1947.

7. MOGI, M. Variation in oviposition, hatch rate and setal morphology in labora tory strains of Aedes albopictus. Mosc. News, 42: 196-211, 1982.

8. ROZEBOOM, L.E. \& BRIDGES, J.R. Relative population densities of Aedes alhopictus and A. guamensis on Guam. Bull. Wld Hlih Org. 46: 477.83, 1972.

9. USINGI:R, R.I. Entomological phases of the recent dengue cpidemic in Honolulu. Puhl. Health Rep., (Wash.), 59:423-30, 1944. 